\title{
3D Printed N-95 Masks During the COVID-19 Pandemic: Lessons Learned
}

\author{
Carole S. L. Spake $1{ }^{1,3}$ Thomas N. Carruthers, ${ }^{2}$ Joseph W. Crozier, ${ }^{1}$ \\ Loree K. Kalliainen, ${ }^{1}$ Reena A. Bhatt, ${ }^{1}$ Scott T. Schmidt, ${ }^{1}$ \\ and Albert S. Woo ${ }^{1}$ \\ ${ }^{1}$ Division of Plastic Surgery, The Warren Alpert Medical School of Brown University, Providence, RI, USA; ${ }^{2}$ Division of \\ Vascular Surgery, The Warren Alpert Medical School of Brown University, Providence, RI, USA; and ${ }^{3}$ Brown University, \\ Box G-9999, 34 Olive Street, Providence, RI 02902, USA
}

(Received 17 June 2021; accepted 20 August 2021; published online 3 September 2021)

Associate Editor Stefan M. Duma oversaw the review of this article.

\begin{abstract}
Early in 2020, the pandemic resulted in an enormous demand for personal protective equipment (PPE), which consists of face masks, face shields, respirators, and gowns. At our institution, at the request of hospital administration, the Lifespan 3D Printing Laboratory spearheaded an initiative to produce reusable N95 masks for use in the hospital setting. Through this article, we seek to detail our experience designing and 3D printing an N95 mask, highlighting the most important lessons learned throughout the process. Foremost among these, we were successful in producing a non-commercial N95 alternative mask which could be used in an era when N95 materials were extremely limited in supply. We identified five key lessons related to design software, 3D printed material airtightness, breathability and humidity dispersal, and ability for communication. By sharing our experience and the most valuable lessons we learned through this process, we hope to provide a helpful foundation for future 3D-printed N95 endeavors.
\end{abstract}

Keywords-Fused deposition modeling (FDM), Additive manufacturing, Personal protective equipment (PPE), Computer Aided Design (CAD).

\section{INTRODUCTION}

The beginning of the COVID-19 Pandemic was characterized by an overwhelming surge in cases with limited knowledge on how to best manage the crisis from an economic and healthcare management perspective. Early in 2020, the pandemic resulted in an

Address correspondence to Carole S. L. Spake, Brown University, Box G-9999, 34 Olive Street, Providence, RI 02902, USA. Electronic mail: carolespake@gmail.com enormous demand for personal protective equipment (PPE), which consists of face masks, face shields, respirators, and gowns. ${ }^{15}$ Indeed, on March 3, 2020, the World Health Organization (WHO) called on governments and industries to increase manufacturing by $40 \%$ to meet the global demand for PPE. ${ }^{29}$ Concerted efforts from various fields and industries were made to address the shortages faced worldwide. For example, research journals issued calls for solutions, production lines were repurposed to manufacture PPE, and Project N95 was founded to identify and redistribute PPE to high-need regions. ${ }^{1,21,26}$

On a more local scale, non-traditional manufacturers contributed to relief efforts within their region through the use of $3 \mathrm{D}$ printing. Over the last year, various PPE, diagnostic, and treatment-related items have been innovated through $3 \mathrm{D}$ design and printing. ${ }^{13}$ A non-exhaustive list of examples includes 3D printed face shields, ${ }^{14,27,28,23}$ nasopharyngeal swabs, ${ }^{2,10}$ mask connectors for continuous positive airway pressure (CPAP) and bilevel positive airway pressure (BiPAP) devices, ${ }^{4}$ and N95 masks. ${ }^{18,19,22,25}$ Beyond 3D printing, collaborative efforts were made to manufacture face cloth coverings ${ }^{11}$ and methods to decrease contact with patients during airway management, such as particle containment chambers. ${ }^{17}$

At our institution, at the request of hospital administration, the Lifespan 3D Printing Laboratory spearheaded an initiative to produce reusable N95 masks for use in the hospital setting. While partially successful, this effort was eventually discontinued 
when the supply of commercially available N95 masks became more readily accessible. Although many 3D Printed N95 mask designs are now accessible through sources such as the NIH Exchange, ${ }^{20}$ many were not available until after we developed our own processes. As a conclusion to this undertaking, we seek to detail our experience designing and 3D printing an N95-style mask, highlighting the most important lessons learned throughout the process.

\section{METHODS}

\section{Case Summary: Our Design Process, Successes, and Failures}

The Lifespan 3D Printing Lab initiated a project to manufacture N95 masks using 3D printing technology. Early in the process, we investigated several opensource designs, including the Montana Mask (https:// www.billingsclinic.com/foundation/3d-printed-surgical -mask/), the S.A.F.E. Mask (https://web.musc.edu/in novation/covid-19-innovation/safe-cartridge-system-a nd-masks), and the NanoHack mask (https://copper3 d.com/hackthepandemic/). ${ }^{18,19,25}$ However, we quickly determined that directly $3 \mathrm{D}$ printing masks would not be feasible for large-scale production, as a single mask required several hours to print. Any effort to print hundreds or thousands of masks would therefore prove impractical in regards to time. Additionally, each of the designs investigated required post-processing procedures (e.g., heating and molding to face), which entailed close contact and would not adhere to social distancing guidelines. Moreover, additional postprocessing time to conform each mask to an individual was deemed impractical on a larger scale when thousands of hospital workers might potentially need to be fitted. As a result, the decision was made to design and $3 \mathrm{D}$ print a mold. Additionally, the team chose to manufacture N95 masks from medical grade silicone, a material with a proven safety record which would be flexible and easily conform to each user's face.

We eventually designed a mold using Fusion 360 software (Autodesk, San Rafael, California) utilizing a cup respirator (3M, Saint Paul, Minnesota) as a starting design reference point while also taking inspiration from the Montana Mask (Spark R\&D, Bozeman, Montana), in which the flexible face mask was separate from the stiff cartridge which would hold N95 material in place. ${ }^{25}$ The final mask design was comprised of two parts: a skin-safe silicone portion using Dragon Skin 30 (Smooth-on, Macungie, Pennsylvania) and a 3D printed filter holder (Fig. 1a). To allow for reusability, an external filter cap was de- signed to protect the reusable N95 material from contamination (Fig. 1b). Two sizes were designed to account for different face shapes. In order to allow for manufacture, the final silicone mold consisted of two parts bolted together, with sites through which silicone could be injected (Fig. 2).

With regard to filters, we were provided an allotment of the duck bill masks (Kimberly-Clark Corporation, Irving, Texas) to use as raw material. Given the unpredictable availability of disposable N95 masks at the time of development, the decision was made to try to optimize the number of filters which could be produced from a single duck bill mask. To maximize usage, we began with filters measuring $2 \times 2 \mathrm{~cm}$, allowing up to 10 filters per source mask (Fig. 3a). However, although this size was utilized in other designs, ${ }^{25}$ we found the small surface area of the filter notably decreased the mask's breathability. Consequently, we gradually expanded the filter size to fashion four filters from a single duck bill mask, resulting in the "Superman"-shaped filter design (Fig. 3b). Both filter sizes were tested using the Bitrex Fit Test Kit (3M, Saint Paul, Minnesota). All of the N95 masks passed fit testing. With the success of testing and FDA emergency use authorization, ${ }^{9}$ we started to immediately scale up production in the interest of providing early access to functional (albeit not ideal) masks available for healthcare providers, as more refined designs continued to be developed. For those interested in the specifics of the mask, the following sections serve to provide further details on the mask and mold design, mask fabrication, assembly, and testing.

\section{Mask and Mold Design}

As aforementioned, the final mask design was predominantly comprised of a silicone portion and a 3D printed filter holder (Fig. 1). The filter holder was designed using SOLIDWORKS 3D CAD (Dassault Systèmes, Vélizy-Villacoublay, France). The shape of the filter holder was determined based on the final design of the filter (Fig. 3b). This resulted in a "Superman"-shaped filter and filter holder. The posterior portion of the filter cap was designed such that it could securely press-fit into the silicone portion of the mask (Fig. 4). The filter holder itself is composed of a body, with a press-fit filter cap (Fig. 5).

The silicone body of the mask was also designed using SOLIDWORKS 3D CAD. The anterior most portion of the mask was designed to securely press-fit into the filter holder (Fig. 4). The posterior portion was scaled in order to create two different mask sizes while maintaining the dimensions for the filter holder attachment. Two loops were added on each side of the 

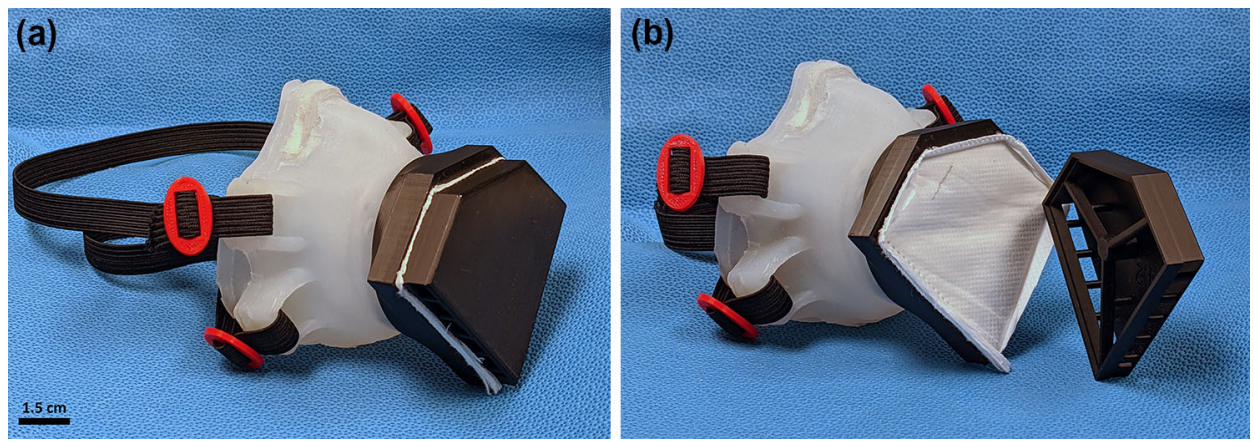

FIGURE 1. (a) Final mask design comprised of silicone portion and 3D printed filter cover. (b) Demonstration of removable filter cap for protection of $\mathrm{N} 95$ material.

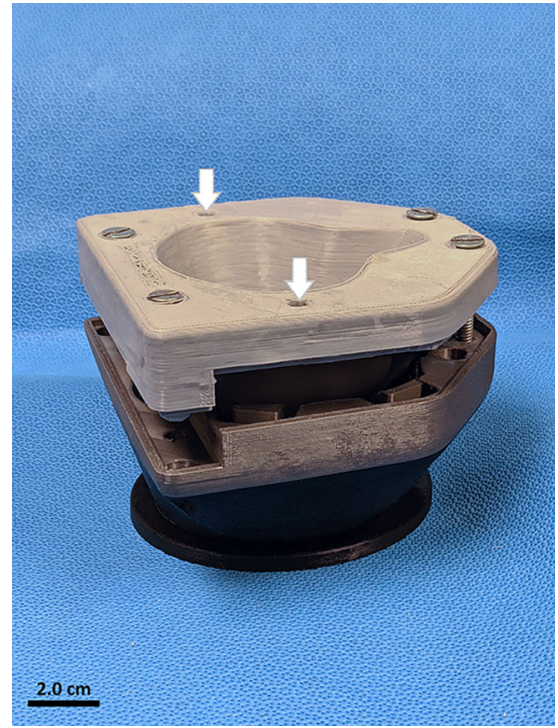

FIGURE 2. Final 3D printed mold for silicone portion of mask. The mold is comprised of two parts which are bolted together. The top half (light grey) of the mold was designed to have sites through which silicone could be injected (white arrows).

mask in order to attach elastics to secure the mask to the face.

Once the mask design was finalized, a two-part negative mold was designed again using SOLIDWORKS 3D CAD (Fig. 2). Four sites for bolt placements were incorporated to prevent silicone leakage during manufacture. Injection sites were designed on the superior aspect of the mold to allow for ease of injection molding and to minimize silicone leakage (Fig. 2).

\section{Mask Fabrication (Molding and Filter Holder Printing)}

Once the final mask mold design was agreed upon, this was manufactured using multiple Prusa i3 MK3S 3D printers with polylactic acid (PLA) filament. Care
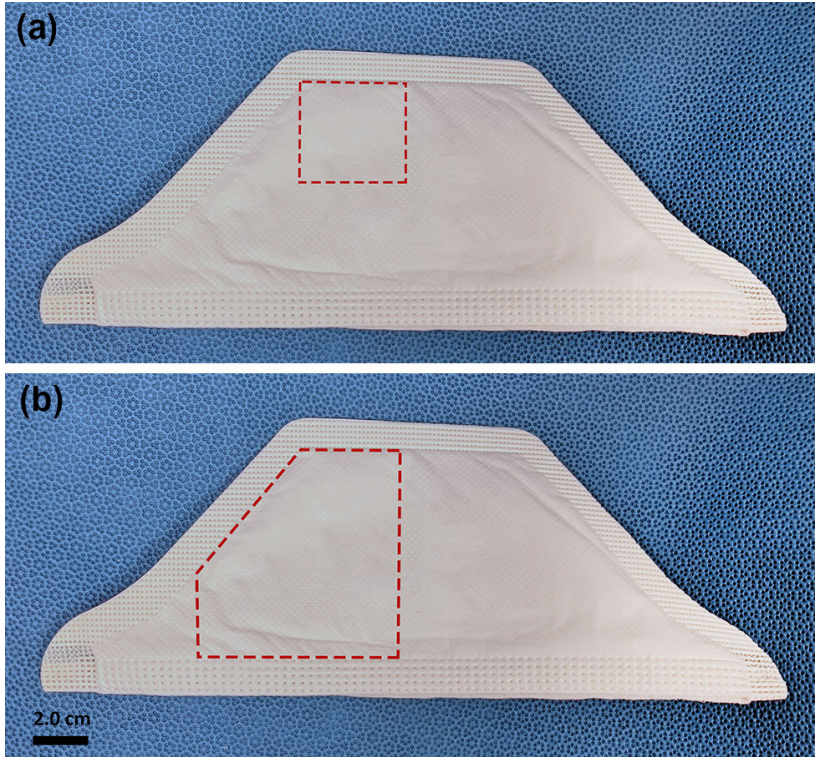

FIGURE 3. (a) Duckbill mask with initial $2 \times 2 \mathrm{~cm}$ filter design, which allowed for 10 filters per source mask (red dashed line). (b) Duckbill mask with the final Supermanshaped filter design (red dashed line), which allowed for 4 filters per mask.

was taken to spray the mold with Ease Release 200 (Smooth-on, Macungie, Pennsylvania) to facilitate removal of the silicone mask from the mold without damage. After the two mold parts were bolted together, a skin-safe silicone portion using Dragon Skin 30 (Smooth-on, Macungie, Pennsylvania) was mixed and injected into the mold. To expedite the speed of silicone curing, molds were placed in an incubator at $60{ }^{\circ} \mathrm{C}$ for a maximum cure time of $30 \mathrm{~min}$.

With regard to the filter holder, a series of experiments were conducted in order to determine the optimal printer settings to prevent air leakage. The goal was to maximize the impermeability of the filter holder, as any permeability would result in less than N95 filtration efficiency. The experiments consisted of placing a filter holder in a water bath and measuring 

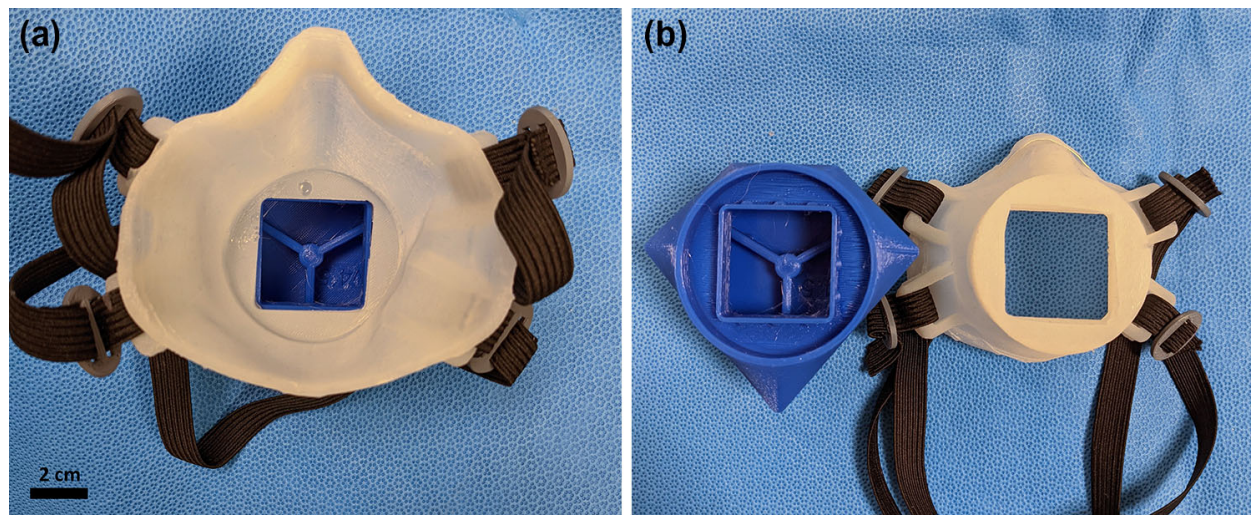

FIGURE 4. (a) Demonstration of silicone mask and filter holder assembled and (b) disassembled.

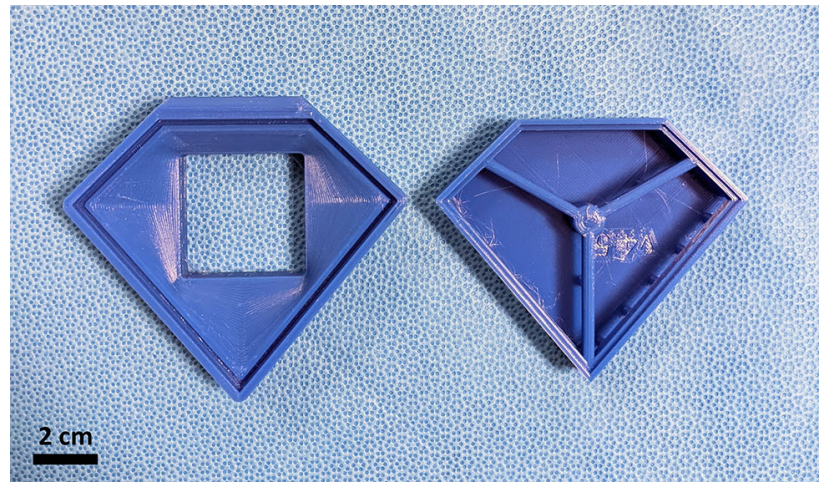

FIGURE 5. Disassembled filter holder with body (left) and filter cap (right).

the time required for 2.4 psi of air to leak out of the material. With each experiment, a different combination of settings was evaluated. The settings investigated included: vertical shells, horizontal shells, infill, overlap, extrusion multiplier, and printer temperature. Ultimately, we determined that increasing the extrusion multiplier, printer temperature, and vertical shells (wall perimeter) resulted in the optimal impermeability while maintaining a reasonable print time.

\section{Final Assembly}

The final assembly of the mask simply requires placing a filter into the filter holder and securing the cap onto the body of the filter holder. The holder is then secured onto the silicone mask using the aforementioned press-fit mechanism. Elastics are then looped through the buckles of the silicone mask, and secured using 3D printed PLA buckles. This allows for adjustment and tightening of the elastics to create a secure seal on the face.

\section{Testing}

All masks underwent standard fit testing using the Bitrex Fit Test Kit. Additionally, the mask design was submitted for official N95 testing by NIOSH. The testing involved both sodium chloride $(\mathrm{NaCl})$ Aerosol testing and inhalation and exhalation resistance testing. These are standardized tests for evaluation of N95 requirements outlined by the Code of Federal Regulations section 42 Part 84 and TEB-APR-STP-0059.

\section{RESULTS}

Throughout this process, our team experienced several successes and failures related to design, manufacturing, and community support. Foremost among these, we were successful in producing a non-commercial N95 alternative mask which could be used in an era when N95 materials were extremely limited in supply. The masks fit most users comfortably with virtually no postprocessing necessary after the components were assembled. Almost all $(95 \%)$ those who passed an initial $30 \mathrm{~s}$ seal check went on to successfully complete Bitrex fit testing, serving as a testament to the excellent seal provided by silicone. All components in contact with skin were comprised of skin-safe materials and true N95 filters were used for air filtration. The mask provided two way protection for both the user (respiratory protection) and surrounding individuals (source control), in case the user was infected. The silicone materials were easily sterilizable with alcohol and the N95 filter was protected from outside contamination with the presence of an external protective shield. Due to the limited contact of the N95 filter with the external environment, the filter could potentially be reused with minimal direct contamination both externally (e.g., blood, sputum) and internally (e.g., skin particulates, saliva, lipstick). We were successful in gathering support from the community, in the form of 
dozens of medical students, faculty, and community members who volunteered their time and resources to produce our end product.

Despite these successes, the mask design had inherent limitations. The silicone material had a tendency to collect humidity, resulting in water collection within the mask when used for extended periods of time. Communication was also noted to be an important issue for clinical providers, negating the practical use of snorkeling masks ${ }^{12}$ for clinical care. Despite reasonable communication ability in face-to-face interactions, our N95 mask was noted to be challenging when trying to communicate over the telephone. The voice was somewhat ruffled, sometimes requiring the operator to raise their voice to make themselves heard in busy or loud environments, such as the operating room. The successes and limitations of our mask design are summarized in Table 1. Regardless, due to overall success of mask design and positive reviews from clinical staff, we proceeded to submit the design for official N95 testing by NIOSH. Unfortunately, while the FDA had processes in place for Emergency Use Authorization and expedited review for products related to management of the COVID Pandemic, NIOSH had no such mechanisms for N95 or other COVID approvals.

Several months later, we received information which suggested that filtration did not achieve the N95 standard $(95 \%$ filtration of particulates less than 0.3 $\mu \mathrm{m})$, but in fact was closer to $89 \%$ filtration. It was determined that this was likely secondary to microscopic leaks within the 3D printed filter holder, which was not sealed to optimize airflow solely through the filter material (see "Lesson 2" section). ${ }^{3}$ Additionally, we learned that the breathability of our mask did not meet the required standards put forth by the FDA, with inhalation resistance at roughly $40 \mathrm{~mm} \mathrm{H}_{2} \mathrm{O}$ (vs. a limit of $35 \mathrm{~mm} \mathrm{H}_{2} \mathrm{O}$ ) and exhalation resistance of 35$40 \mathrm{~mm} \mathrm{H}_{2} \mathrm{O}$ (rather than a limit of $25 \mathrm{~mm} \mathrm{H}_{2} \mathrm{O}$ ). The second issue proved much more challenging, as improved breathability would necessitate use of larger
N95 filter material, necessitating a complete redesign of the filter holder. The results of the breathability and filtration testing performed according to the Code of Federal Regulations can be found in Table 2. Based on these results and the resolution of the PPE crisis at our local facilities, we chose not to proceed further with the $3 \mathrm{D}$ printed N95 project, as there was less institutional support to do so.

Through this experience, we developed an understanding of the infrastructure required to rapidly $3 \mathrm{D}$ design and print N95 masks. We learned a number of different lessons that we would like to share with others, should they be interested in pursuing a similar effort in the future. From our successes and failures throughout this process, we have identified key design elements that must be considered when 3D printing an N95 mask. The second half of this article is dedicated to discussing the design elements, as well as identifying and detailing five crucial lessons we feel are valuable for success.

\section{DISCUSSION}

\section{Key Considerations for Mask Design}

Our experience allowed us to identify several essential considerations when designing and manufacturing an N95 mask using 3D printing technology, particularly for use in a medical setting. Broadly, the essential considerations encompass functional, practical, and manufacturing aspects of the design. First and foremost, the mask must fit a variety of face sizes and shapes. To achieve this end, more than one size may need to be offered. In addition, the design must provide an adequate seal on the face utilizing skin safe materials. It is also important to consider both the intended filter material and the need to offer two-way protection (i.e., no exhalation valve). The latter is an essential element of design, as source control is a key consideration in preventing transmission in both directions, particularly in the healthcare environment. While

TABLE 1. Summary of pertinent positive and negative characteristics of key mask components.

\begin{tabular}{|c|c|c|}
\hline $\begin{array}{l}\text { Mask compo- } \\
\text { nent }\end{array}$ & Positive & Negative \\
\hline $\begin{array}{l}\text { FDM printed } \\
\text { filter holder }\end{array}$ & $\begin{array}{l}\text { Easy and quick to manufacture, design prevents contamination of } \\
\text { N95 filter material }\end{array}$ & $\begin{array}{l}\text { Permeable materials hinders true N95 filtering capa- } \\
\text { bilities }\end{array}$ \\
\hline $\begin{array}{l}\text { N95 filter } \\
\quad \text { measuring } \\
4 \times 4 \mathrm{~cm}\end{array}$ & Uses proven N95 Duck Bill mask as raw material for filter & $\begin{array}{l}\text { Small filter size hinders breathability and communi- } \\
\text { cation }\end{array}$ \\
\hline Silicone mask & $\begin{array}{l}\text { Provides adequate seal, fits variety of faces in only two sizes, skin- } \\
\text { safe material, comfortable, transmits sound well in face-to-face } \\
\text { interaction, easy to sterilize with alcohol }\end{array}$ & $\begin{array}{l}\text { Poor breathability, retains humidity, requires } 3 \mathrm{D} \\
\text { printed injection mold, poor sound transmission } \\
\text { over telephone }\end{array}$ \\
\hline
\end{tabular}

FDM fused deposition modelling. 
TABLE 2. Results of outside laboratory testing of mask for breathability and filtration in accordance with 42 CFR Part 84 .

(a) Breathability-inhalation and exhalation resistance testing

\begin{tabular}{lll}
\hline Test article & Inhalation resistance $\left(\mathrm{mm} \mathrm{H}_{2} \mathrm{O}\right)$ & Exhalation resistance $\left(\mathrm{mm} \mathrm{H}_{2} \mathrm{O}\right)$ \\
\hline $\mathrm{RIH} 21$ & 18.2 & 31.0 \\
$\mathrm{RIH} 22$ & 49.5 & 45.1 \\
$\mathrm{RIH} 23$ & 39.2 & 35.8
\end{tabular}

(b) Filtration-sodium chloride $(\mathrm{NaCl})$ aerosol testing

\begin{tabular}{llll} 
Test article & Corrected initial airflow resistance $\left(\mathrm{mm} \mathrm{H}_{2} \mathrm{O}\right)$ & Maximum particle penetration $(\%)$ & Filtration efficiency $(\%)$ \\
\hline $\mathrm{RIH} 1$ & 74.0 & 10.9 & 89.1 \\
$\mathrm{RIH} 2$ & 77.4 & 11.3 & 88.7 \\
$\mathrm{RIH3}$ & 73.5 & 12.8 & 87.2 \\
\hline
\end{tabular}

(a) Inhalation resistance criteria stated in 42 CFE Part 84.180: initial inhalation not exceeding $35 \mathrm{~mm}$ water column height pressure. Exhalation resistance criteria stated in 42 CFR Part 84.180: initial exhalation not exceeding $25 \mathrm{~mm}$ water column height pressure. The test articles submitted do not conform to this NIOSH criterion for airflow resistance.

(b) The NIOSH N95 filter efficiency as stated in 42 CFR Part 85.181 is a minimum efficiency for each filter of $\geq 95 \%$ ( $\leq 5 \%$ penetration). The test articles submitted do not conform to the NIOSH N95 criteria for filter efficiency.

masks with exhalation valves may be more comfortable, the air exhaled by the wearer is not filtered. As a result, additional precautions must be employed while wearing such masks. With regard to practicality, the mask must be adequately breathable to ensure comfort during long periods of wear, possibly for $8 \mathrm{~h}$ at a time (a standard hospital shift). Similarly, the mask must be designed in a manner that allows for easy transmission of speech, so that the caregiver's ability to communicate is unfettered. Lastly, from a manufacturing standpoint, while 3D printing is an excellent and costefficient means of producing high quality prototypes, production in large volumes may be most cost-effective and more efficient utilizing standard manufacturing processes.

\section{Lesson 1: Use CAD, Not Blender}

Early in our design process, we used Blender software (The Blender Foundation, Amsterdam, The Netherlands) to design the original molds. Blender is an open-source 3D software which supports the full gamut of 3D creation, including (but not limited to) modeling, animation, simulation, rendering, and game creation. The decision to use Blender was based on previously established expertise with the software package and ability to easily reverse engineer the shape of a standard cup N95 mask with scanning technologies. However, we quickly realized that Blender is not appropriate for iterative design changes. The process of designing the final mold was lengthy and required many iterations based on problems and potential pitfalls we noted on successive prototypes.
Notably, the final mask design resulted in two mask sizes to better fit various face shapes. This required scaling the posterior portion of the mask while maintaining the size of the anterior portion to press fit securely with the filter holder. While scaling entire objects is achievable in Blender, it is much more difficult to successfully scale segments of designs (e.g., only the posterior portion of the mask). These changes were not simple to implement on Blender, as the software does not allow for easy parametric modelling. While possible, each change required several hours to effectuate, rather than minutes on Computer Aided Design (CAD) software. Additionally, we realized that a CAD design would be more amenable than a Blender file to scaling our operation to mass production through outsourcing the manufacturing to a third party. Consequently, we transitioned to the CAD software SOLIDWORKS 3D CAD.

Ultimately, the difficulty and challenges presented through the use of Blender reinforced that these two software modalities are not intended for the same purpose. While Blender is designed to create $3 \mathrm{D}$ art, animations, and visual effects, it is not well suited for engineering designs. On the other hand, not only is CAD software well-suited for $3 \mathrm{D}$ printed objects, it also was created with the purpose to serve as an engineering platform for iterative design. ${ }^{7}$ Reverse engineering a product based on imaging is more difficult, however, as scanned shapes are not easily reduced to CAD design elements. As such, we learned that the use of CAD software allows for more exact modeling, which proved invaluable during subsequent design changes. 


\section{Lesson 2: FDM Materials Are Not Impermeable}

One of the important considerations when 3D printing N95 masks is the porosity of the materials used. The vast majority of open-source 3D printed N95 mask designs employ fused deposition modeling (FDM) or fused filament fabrication (FFF) printing technology. Essentially, this 3D printing process uses a continuous thermoplastic material filament that is heated through a printer extruder head and deposited onto the surface of the growing print. The intricacies of FDM 3D printing are beyond the scope of this paper. However, it is important to note that the materials used are not completely airtight. ${ }^{3}$

While designing our 3D printed filter holder, we sought to achieve the highest degree of impermeability to prevent air leaks through the material. We achieved this by conducting a series of experiments testing combinations of different materials and infills patterns. During the experiments, the filter holder was placed in a water bath and air was pumped into the holder. Our experiments allowed us to identify a combination of printer settings and materials that we felt would provide a reasonable level of impermeability to air.

Ultimately, we learned that there is a fine line between achieving an acceptable degree of impermeability while also maintaining speed of production and keeping the cost of the filter holder within reason. On one hand, the infill could be increased to $100 \%$ (making it a solid object), as this would decrease the porosity of the filter holder and make it more airtight. However, increasing the infill would also drastically increase the time and material required to print a single filter holder, while also increasing the total weight of the mask. Alternatively, another solution to eliminate permeability is to seal the 3D printed parts of the mask. While several methods to seal 3D printed channels have been described, this process is laborious and may not be feasible for large scale production of filter holders for N95 masks. ${ }^{3}$ Overall, these constraints would make achieving impermeability and thus true N95 filtration difficult in a mask that is solely $3 \mathrm{D}$ printed. Therefore, if sealing is not possible, a more realistic option may instead be to manufacture the mask by injection molding or to print the device utilizing other technologies (e.g., stereolithography). Alternatively, other printing processes that can manufacturing impermeable materials, such as selective laser sintering (SLS), may be considered. However, this type of printing process is not as accessible and as widespread as FDM. As our process and output required the use of many FDM printers through community volunteers, using alternate printing processes was not feasible.
While the majority of our mask was manufactured from silicone, we ultimately believe that the porosity of FDM materials used for the 3D printed filter holder may have contributed to our mask's failure to achieve $95 \%$ filtration during third party testing. As a result, we feel that the degree of porosity inherent to FDM $3 \mathrm{D}$ printed materials must be given great consideration when undertaking the task of 3D printing an N95 mask.

\section{Lesson 3: 3D Printing Cannot Provide an Appropriate Filter}

While this may be common sense to some, it bears noting that although $3 \mathrm{D}$ printing technology can be used to manufacture masks, it cannot be used to 3D print N95 grade filters. As a result, all designs must take this limitation into account and the configuration must allow for attachment of an effective filter. Thus, the ability to successfully 3D print an N95 mask is predicated on the ability to acquire filters that can be refashioned to fit into the mask, as no additive manufacturing process can match this. As previously explained, we were provided an allotment of duck bill masks, which we used as raw material for filters. Aside from true N95 material, high efficiency particular air (HEPA) filters may be a viable alternate option. In order to quality as HEPA grade, a filter must remove a minimum of $99.97 \%$ of particles measuring between 0.15 and $0.2 \mu \mathrm{m} .{ }^{5,16}$ This filtering efficacy requirement exceeds the standards set for achieving N95 capability. Additionally, HEPA filters have been demonstrated to have similar breathability as standard N95 respirators. $^{24}$

Other high-grade filters used during the height of the pandemic included those found in commercial and household products (e.g., air-conditioning filters). However, it is important to note that the minimum efficiency reporting value (MERV) rating for such filters typically ranges 13 to 14 , signifying a reduction of the flow of particles larger than $0.3 \mu \mathrm{m}$ by 50 or $70 \%$, respectively. Conversely, the MERV rating of N95 filters is 16 , which signifies a reduction in particle flow by $95 \% .{ }^{15}$ Therefore, while filters from commercial and household products may be readily available, these filters are unlikely to achieve true N95 filtering capabilities. For reasonable adoption in the hospital setting, filter material must meet or exceed N95 standards.

\section{Lesson 4: Consider Breathability and Humidity}

One of the other weaknesses of the aforementioned open-source 3D printed masks centers on the issue of breathability. We found that generally, the open- 
source designs only allowed for placement of very small filters on the 3D printed mask. Since the mask is sealed to the face, the only area through which air should penetrate is through the filter. As previously discussed, smaller filters impede the user's ability to breathe comfortably while using the N95 mask. As a result, we realized that the filter area had to be large enough to permit an acceptable level of breathability. This is inversely proportional to the number of filters which can be sourced from a single duckbill mask.

Despite our own attempts to incorporate improved breathability into our design, we discovered after testing that this issue remained a critical failure, with higher than acceptable inspiration and exhalation resistance values. The specific requirements for passing this criteria are outlined by the Code of Federal Regulations section 42 Part $84.180 .{ }^{8}$ While we ultimately increased our filter size to maximize breathability, third party testing results indicated that our filter was still not large enough to meet the FDA's airflow resistance criteria. Based on these requirements, we determined that no more than two filters could be harvested from a single duckbill mask, as we had previously maximized the surface area available when a single donor mask was divided into four parts.

On a similar note, the issue of humidity retention inside the mask must be addressed. Any non-breathing material used for a mask (e.g., silicone or plastic shells) will result in accumulation of humidity inside the mask, which may affect the seal and lead to user discomfort. While we chose not to pursue further design iterations to address this problem, we believe there are potential solutions to preventing humidity retention. For example, our proposal of a larger filter size would serve the added benefit of reducing humidity deposition by increasing airflow exchange. Additionally, a collection gutter or condensation retrieval mechanism could be incorporated into the mask to assist in removing excess humidity. Alternatively, an air desiccant (e.g., Nafion tubing) may be added to the mask and replaced after each use to efficiently remove water from the exhaled air. Overall, an important lesson to glean from this experience is that any 3D-printed N95 mask design must consider the breathability standards set forth by the FDA, while also addressing user comfort from a humidity-evacuation perspective.

\section{Lesson 5: Ability to Communicate is Critical}

One of the key failures with most of the mask designs we evaluated revolves around the issue of communication. The ability to verbally interact with a patient is a critical element of a clinician's job; in addition to protecting the user, the mask must allow for effective transmission of speech. If a patient or colleague cannot hear a physician through their N95 mask, this mask is not a viable option. Indeed, this is a key reason why reusable elastomeric masks or even full-face respirators (i.e., traditional gas masks or work masks used by painters) were not largely adopted by hospitals.

One of the designs we had initially considered was a 3D printed adapter for a snorkel mask, such as the made commercially available by $3 \mathrm{D}$ Chimera. ${ }^{6}$ The adapter mounts to a snorkel mask and couples to a standard filter, thereby fashioning an N95 mask from an easily purchasable item. While a plausible and creative solution, the snorkel mask did not transmit sound to permit effective communication. We found that the issue of maintaining acceptable speech transmission was a recurring problem that many did not anticipate. While we did not pursue further avenues to improve communication in masks with poor speech transmission, a potential solution may involve incorporation of devices to amplify sound, such as microphones. However, such modifications would increase the complexity of the mask design and manufacturing process.

Indeed, the ability to communicate not only with patients but with other clinicians and staff is critical to the function of clinical providers. This requirement cannot be underestimated in the design of appropriate N95 level PPE.

\section{CONCLUSION}

Overall, the COVID-19 Pandemic presented many unprecedented challenges to the global community, including a global PPE shortage. Our 3D Printing Laboratory sought to contribute to the local relief efforts by providing 3D printing N95 masks. However, this was not without considerable obstacles. We identified five key lessons related to design software, 3D printed material airtightness, breathability and humidity dispersal, and ability for communication. By sharing our experience and the most valuable lessons we learned through this process, we hope to provide a helpful foundation for future 3D-printed N95 endeavors.

\section{ACKNOWLEDGEMENTS}

The authors wish to thank the following persons and groups, without whom this endeavour would not have been possible: Professor Christopher Bull and the Brown University Design Workshop, Leo Salvaggio 
and Kelly Egan, Katherine Westrom, Ronald Akiki, the Lifespan CMO Office, Brown University medical students and Rhode Island community volunteers.

\section{CONFLICT OF INTEREST}

The authors have no conflicts of interests of financial disclosures to disclose.

\section{REFERENCES}

${ }^{1}$ Bauchner, H., P. B. Fontanarosa, and E. H. Livingston. Conserving supply of personal protective equipment-a call for ideas. JAMA 2020. https://doi.org/10.1001/jama. 2020.4770.

${ }^{2}$ Callahan, C. J., R. Lee, K. E. Zulauf, L. Tamburello, K. P. Smith, J. Previtera, A. Cheng, A. Green, A. A. Azim, A. Yano, N. Doraiswami, J. E. Kirby, and R. A. Arnaout. Open development and clinical validation of multiple 3Dprinted sample-collection swabs: rapid resolution of a critical COVID-19 testing bottleneck. medRxiv 2020. http s://doi.org/10.1101/2020.04.14.20065094.

${ }^{3}$ Carrell, C. S., C. P. McCord, R. M. Wydallis, and C. S. Henry. Sealing 3D-printed parts to poly(dimethylsiloxane) for simple fabrication of microfluidic devices. Anal. Chim. Acta 2020. https://doi.org/10.1016/j.aca.2020.05.014. .

${ }^{4}$ Cavallo, L., A. Marcianò, M. Cicciù, and G. Oteri. 3D printing beyond dentistry during COVID 19 epidemic: a technical note for producing connectors to breathing devices. Prosthesis 2:46-52, 2020.

${ }^{5}$ Christopherson, D. A., W. C. Yao, M. Lu, R. Vijayakumar, and A. R. Sedaghat. High-efficiency particulate air filters in the era of COVID-19: function and efficacy. Otolaryngol. Head Neck Surg. (U.S.) 163:1153-1155, 2020. .

63D Chimera. COVID-19 3D Printed Snorkel Adapter. 3D Chimera, 2020. https://3dchimera.com/products/covid-193d-printed-snorkel-adapter.

${ }^{7}$ Ekströmer, P., and R. Wever. "Ah, I see what you didn't mean" exploring Computer Aided Design tools for design ideation. Des. J. 22:1883-1897, 2019. .

${ }^{8}$ Electronic Code of Federal Regulations (eCFR). https:// www.ecfr.gov/cgi-bin/text-idx?node = se42.1.84_1180\&rgn $=\operatorname{div} 8$.

${ }^{9}$ Food and Drug Administration. Coronavirus Disease 2019 (COVID-19) Emergency Use Authorizations for Medical Devices. Food and Drug Administration, 2020. https://w ww.fda.gov/medical-devices/emergency-use-authorization s-medical-devices/coronavirus-disease-2019-covid-19-emer gency-use-authorizations-medical-devices.

${ }^{10}$ Ford, J., T. Goldstein, S. Trahan, A. Neuwirth, K. Tatoris, and S. Decker. A 3D-printed nasopharyngeal swab for COVID-19 diagnostic testing. 3D Print. Med. 6:1-7, 2020. .

${ }^{11}$ George, M. P., L. A. Maier, S. Kasperbauer, J. Eddy, A. S. Mayer, and C. M. Magin. How to leverage collaborations between the BME community and local hospitals to address critical personal protective equipment shortages during the COVID-19 Pandemic. Ann. Biomed. Eng. 48:2281-2284, 2020. .

${ }^{12}$ Kechli, M. K., J. Lerman, M. M. Ross, and Modifying a full-face snorkel mask to meet N95 respirator standards for use with coronavirus 2019 disease, . patients. A\&A Pract. 2020. https://doi.org/10.1213/XAA.0000000000001237.

${ }^{13}$ Korr, M. Q\&A with Albert S. Woo, MD: forging a new frontier in 3D printing during COVID-19 Pandemic. R. $I$. Med. J. 103:74-75, 2020.

${ }^{14}$ Kursat Celik, H., O. Kose, M. E. Ulmeanu, A. E. W. Rennie, T. N. Abram, and I. Akinci. Design and additive manufacturing of medical face shield for healthcare workers battling coronavirus (COVID-19). Int. J. Bioprint. 6:1$21,2020$.

${ }^{15}$ Livingston, E., A. Desai, and M. Berkwits. Preventing the spread of infection within the hospital setting depends on proper use of personal protective equipment (PPE). JAMA 323:1912-1914, 2020.

${ }^{16}$ Malhotra, V. A low-cost solution for retrofitment of HEPA filter in healthcare facilities providing care to COVID-19 patients. Indian Pediatr. 57:477, 2020.

${ }^{17}$ Maloney, L. M., A. H. Yang, R. A. Princi, A. J. Eichert, D. R. Hébert, T. V. Kupec, A. E. Mertz, R. Vasyltsiv, T. M. Vijaya Kumar, G. J. Walker, E. J. Peralta, J. L. Hoffman, W. Yin, and C. R. Page. A COVID-19 airway management innovation with pragmatic efficacy evaluation: the patient particle containment chamber. Ann. Biomed. Eng. 48:23712376, 2020. .

${ }^{18}$ Medical University of South Carolina. S.A.F.E. Cartridge System and Masks. Medical University of South Carolina, 2020. https://web.musc.edu/innovation/covid-19-innovatio $\mathrm{n} /$ safe-cartridge-system-and-masks.

${ }^{19}$ National Institutes of Health. NanoHack Mask | NIH 3D Print Exchange. National Institutes of Health, 2020. http s://3dprint.nih.gov/discover/3dpx-013388.

${ }^{20}$ National Institutes of Health. Mask NIH 3D Print Exchange. National Institutes of Health, 2020. https://3dpri nt.nih.gov/discover/mask.

${ }^{21}$ Project N95. About | Project N95. 2020. https://www.proj ectn95.org/about.

${ }^{22}$ Provenzano, D., Y. J. Rao, K. Mitic, S. N. Obaid, D. Pierce, J. Huckenpahler, J. Berger, S. Goyal, and M. H. Loew. Rapid prototyping of reusable 3D-printed N95 equivalent respirators at the George Washington University. Preprints 1-9, 2020. https://doi.org/10.20944/preprint s202003.0444.v1.

${ }^{23}$ Prusa Face Shield by Prusa Research | Download free STL model | PrusaPrinters. https://www.prusaprinters.org/prin ts/25857-protective-face-shield-rc1.

${ }^{24}$ Saggese, N. P., A. L. Rose, K. Murtagh, A. P. Marks, and V. A. Cardo. An interim solution to the decreased availability of respirators against COVID-19. Anesth. Analg. 2020. https://doi.org/10.1213/ANE.0000000000004879. .

${ }^{25}$ Spark R\&D. Spark R\&D Montana Mask Information Page. Spark R\&D, 2020. https://www.sparkrandd.com/spa rk-rd-montana-mask-information-page/.

${ }^{26}$ Supply Chain Dive. Tracking US Manufacturers' Shift to PPE During the Coronavirus Pandemic. Supply Chain Dive, 2020. https://www.supplychaindive.com/news/us-ma nufacturers-ppe-coronavirus-pandemic/576665/.

${ }^{27}$ Throckmorton, A. L., E. J. Bass, B. Ferrick, A. Ramakrishnan, S. Eichmann, N. Catucci, B. Eshelman, J. McNamara, E. Sundquist, N. Beatson, M. Hirschhorn, P. Menon, E. Datner, R. Stevens, and M. Marcolongo. A cross university-led COVID-19 rapid-response effort: design, build, and distribute Drexel AJFlex face shields. Ann. Biomed. Eng. 49:950-958, 2021.

${ }^{28}$ Wierzbicki, J., M. Nowacki, M. Chrzanowska, R. Matkowski, M. Ziętek, K. Nowacka, A. Maciejczyk, and E. 
Pawlak-Adamska. Additive manufacturing technologies enabling rapid and interventional production of protective face shields and masks during the COVID-19 pandemic. Adv. Clin. Exp. Med. 29:1021-1028, 2020. .

${ }^{29}$ World Health Organization. Shortage of Personal Protective Equipment Endangering Health Workers Worldwide. World Health Organization, 2020. https://www.who.int/ne ws/item/03-03-2020-shortage-of-personal-protective-equip ment-endangering-health-workers-worldwide.

Publisher's Note Springer Nature remains neutral with regard to jurisdictional claims in published maps and institutional affiliations. 